
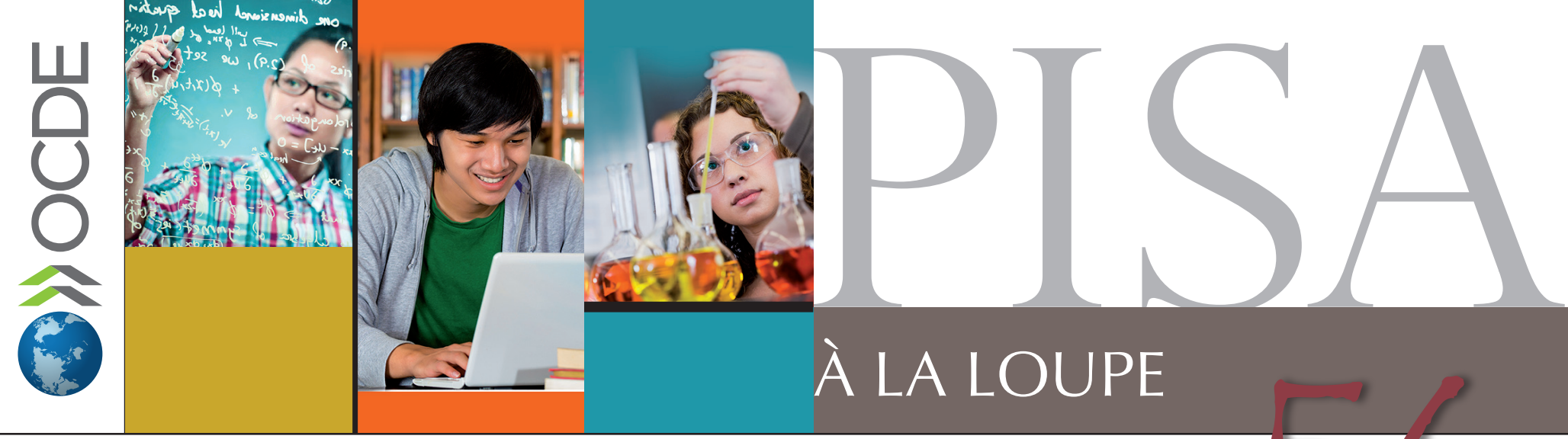

éducation données éducation chiffres éducation politiques éducation analyses éducation statistiques éducation donnée

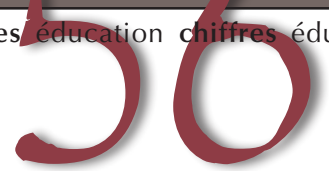

\title{
Quelle confiance les élèves ont-ils en leur capacité à résoudre des problèmes de mathématiques?
}

- En moyenne, dans les pays de l'OCDE, la confiance des élèves en leur capacité à résoudre des problèmes de mathématiques (leur efficacité perçue en mathématiques) est associée à une différence de 49 points de score dans cette matière - soit l'équivalent d'une année de scolarité.

- Il existe une corrélation étroite entre d’un côté, la confiance des élèves en leur capacité à résoudre des problèmes de mathématiques pures et appliquées, et de l'autre, le fait qu'ils aient déjà été exposés ou non à des tầches similaires en classe.

- Lorsque l'on compare des élèves présentant des résultats scolaires et un niveau socio-économique similaires, ceux dont les parents aspirent à ce qu'ils suivent des études supérieures font généralement part d'un niveau plus élevé d'efficacité perçue en mathématiques que ceux dont les parents ne nourrissent pas des attentes aussi élevées à leur égard.

La notion d' « efficacité perçue » renvoie à la conviction qu'ont les élèves de pouvoir, par l'entremise de leurs actes, produire les effets qu'ils escomptent. Cette conviction nourrit leur motivation à agir ou à persévérer face aux difficultés. En 2012, l'enquête PISA a analysé les déclarations des élèves concernant leur efficacité perçue en mathématiques, c'est-à-dire la mesure dans laquelle ils s'estiment capables de résoudre les problèmes de mathématiques qu'ils rencontrent.

L'enquête PISA 2012 a ainsi demandé aux élèves d'indiquer dans quelle mesure ils se sentiraient sûrs d'arriver à mener à bien une série de tâches de mathématiques pures et appliquées, notamment : utiliser un horaire de trains pour calculer combien de temps prendrait le trajet d'un endroit à un autre ; calculer de combien diminuerait le prix d'un poste de télévision après une réduction de $30 \%$; calculer combien de mètres carrés de dalles il faut pour carreler un sol ; calculer la consommation d'essence d'une voiture ; comprendre les graphiques présentés dans les journaux ; calculer la distance réelle entre deux endroits sur une carte à l'échelle 1/10 000 ; et résoudre des équations du type $3 x+5=17$ et $2(x+3)=(x+3)(x-3)$.

Il existe une corrélation étroite entre l'efficacité perçue en mathématiques et la performance dans cette matière.

Au niveau des pays/économies, on observe une corrélation étroite entre l'efficacité perçue en mathématiques et la performance dans cette matière. Les pays présentant une performance moyenne plus élevée en mathématiques sont ceux où les élèves sont plus susceptibles d'indiquer se sentir sûrs d'arriver à résoudre une série de problèmes de mathématiques pures et appliquées. Une corrélation positive s'observe également au sein même des pays. Les élèves faisant part $d^{\prime} u n$ niveau inférieur d'efficacité perçue en mathématiques obtiennent de moins bons résultats dans cette matière que ceux qui indiquent avoir confiance en leur capacité à résoudre des problèmes de mathématiques. En moyenne, dans les pays de l'OCDE, l'efficacité perçue en mathématiques est associée à une différence de 49 points de score dans cette matière - soit l'équivalent d'une année de scolarité. Dans 23 pays et économies, la différence de score en mathématiques associée à l'efficacité perçue des élèves dans cette matière représente 50 points, voire davantage ; au Liechtenstein, au Taipei chinois et au Viêtnam, cette différence atteint au moins 60 points. 
La corrélation entre l'efficacité perçue en mathématiques et la performance dans cette matière se renforce mutuellement. Tandis qu'une meilleure performance en mathématiques entraîne un niveau supérieur d'efficacité perçue, les élèves présentant un niveau inférieur d'efficacité perçue en mathématiques sont quant à eux plus susceptibles d'être peu performants dans cette matière, indépendamment de leurs capacités réelles. Si les élèves ne croient pas en leur capacité à mener à bien certaines tâches, ils ne déploieront pas les efforts nécessaires pour y parvenir ; un niveau insuffisant d'efficacité perçue devient alors une prophétie auto-réalisatrice.

\section{La confiance des élèves en leur capacité à résoudre des problèmes de mathématiques est liée à la fréquence à laquelle ils ont été exposés à des tâches similaires à l'école}

Pourcentage d'élèves déclarant se sentir " sûrs » ou " tout à fait sûrs » d'arriver à calculer la distance réelle entre deux endroits sur une carte à l'échelle 1/10 000 s'ils ont déjà été exposés à ce type de tâche...

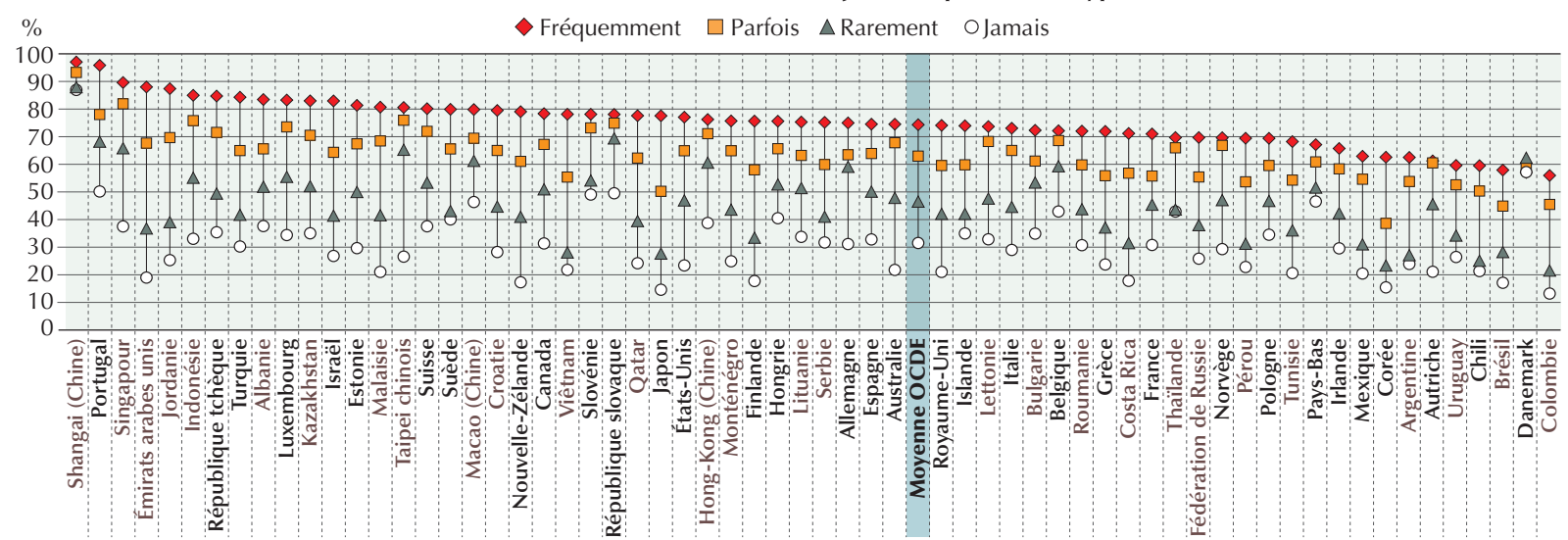

Pourcentage d'élèves déclarant se sentir " sûrs " ou « tout à fait sûrs " d'arriver à résoudre une équation du type $3 x+5=17$ s'ils ont déjà été exposés à ce type de tâche...

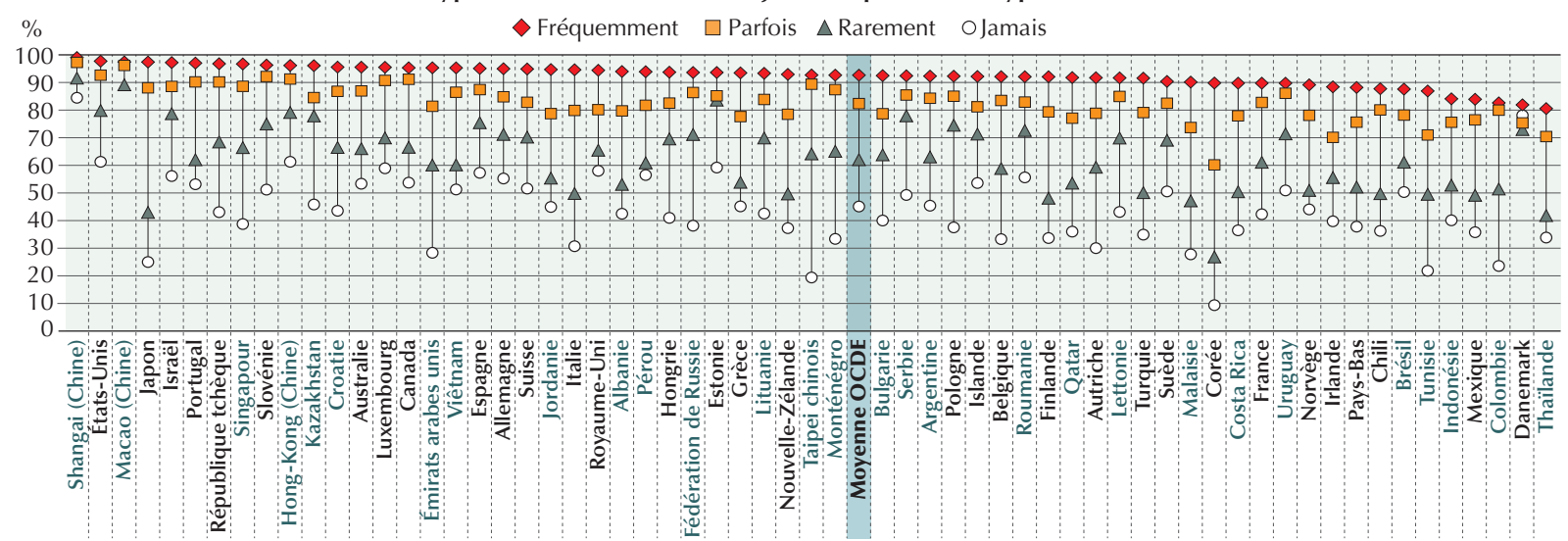

Les pays et économies sont classés par ordre décroissant du pourcentage d'élèves déclarant se sentir "sûrs " ou " tout à fait sûrs " d'arriver à résoudre un problème lorsqu'ils ont déjà été fréquemment exposés à ce type de tâche à l'école.

Source : OCDE, Base de données PISA 2012, tableau III.5.12.

StatLink 交政 http://dx.doi.org/10.1787/888932963996

L'école peut renforcer l'efficacité perçue des élèves en mathématiques

en les exposant à des types variés de tầches...

La corrélation étroite et se renforçant mutuellement entre l'efficacité perçue en mathématiques et la performance dans cette matière laisse penser que les systèmes d'éducation qui favorisent le renforcement de la confiance et de la motivation de leur élèves les aident également à développer certaines compétences spécifiques. L'enquête PISA révèle en effet l'existence d'une forte corrélation entre d'une part, le niveau de confiance qu'ont les élèves en leur capacité à résoudre des problèmes spécifiques de mathématiques pures et appliquées, et d'autre part, le fait qu'ils aient déjà été exposés ou non à des problèmes similaires en classe. Ainsi, bien que $56 \%$ des élèves se sentent sûrs ou tout à fait 
